\title{
Design and Implementation of the Intelligent System for Quantitative Analysis of Sports Motion
}

\author{
Wang Ligang*
}

Chang'an University, China

\begin{abstract}
The paper primarily presents design and implementation methods for the intelligent system for quantitative analysis of sports motion together with the representation methods for human body hierarchy model and action characteristic as well as structure and learning method for matching membership function and fuzzy neural network for pattern recognition of sports motion based on practical situation so as to make relevant personnel better carry out system design and implement system superiority and function by the methods and enable them to offer better services.
\end{abstract}

Keywords: Intelligent system, quantitative analysis of sports motion, system design and implementation.

\section{INTRODUCTION}

Sports motion is the core of sports exercise, in order to further improve sports level, relevant organizations adopt advanced means to better analyze the motion of athlete and determine more standard motion and further standardize the motion of athlete. Relevant personnel carry out management and processing for sports motion images by computer technology for comparison and analysis later and summarize the data on motion characteristic from those images and then build a favorable basis for design and implementation of intelligent system.

During analysis on sports motion images, relevant personnel shall understand two following points: the first is that the human motion is a complex and multi-element issue subject to restrictions of many exercise conditions; the second is that human motion image is body of correlation of multiframe images, characterized by many pictures, affected by the interference factors such as lighting, venue, etc. as well as secondary factors athlete stature and weight. In order to solve the problem, relevant personnel shall design proper intelligent system and ensure its implementation, and bring fuzzy mathematic technology and neural network technology into full play, so as to adopt effective method to remove the interference factor and secondary factors in sports motion and highlight fuzzy characteristic. In the process of adopting neural network technology, relevant personnel shall understand neural network function in its entirety to extract fuzzy rule from sports motion images so as to make intelligent system have better adaptability and analysis capability of sports motion.

\section{CHARACTERISTIC REPRESENTATION METHOD FOR SPORTS MOTION BASED ON FUZZY MATHE- MATIC THEORY}

\subsection{Human Body Hierarchy Frame Structure Model}

Limbs are motion neuron of human body and joint is axis of limb motion, the hierarchy between joints [1] is as shown in the following figure.

It is observed from Fig. (1) that the hierarchical correlation between limbs lies in inheritance of motion and extent of influence of limb motion on entire body, which may be indicated by weighting coefficient of limb motion intensity. Limbs are connected from top to bottom according to hierarchical relation [2]. There is minimum rectangle shown in human motion neuron hierarchical chart, in which the joint is defined as center of the rectangle, with root limb abstracted and indicated by directed line segments along 6 directions from center, namely up, down, left and right with a length of $1 / 2$ unit and other limbs abstracted and indicated by directed line segments from joint with a length of 1 unit. The mathematical representation for hierarchical structure model of limbs in human motion is:

Human body [limb set, joint position set, coupling relation set, motion intensity weighting coefficient set, motion constraint set]

\subsection{Characteristic Representation Method For Sports Motion}

The limb in sports motion image can be split into three types by fuzzy mathematical method, the first is motion characteristic limb set composed of limbs with a large amount of motion; the second is state characteristic limb set composed of limbs with almost no motion but significant influence on motion [3]; the third is fuzzy characteristic limb set composed of limbs ignored or without distinctive characteristics, the mathematical representation for sports motion $\mathrm{Z}(\mathrm{M})$ : 


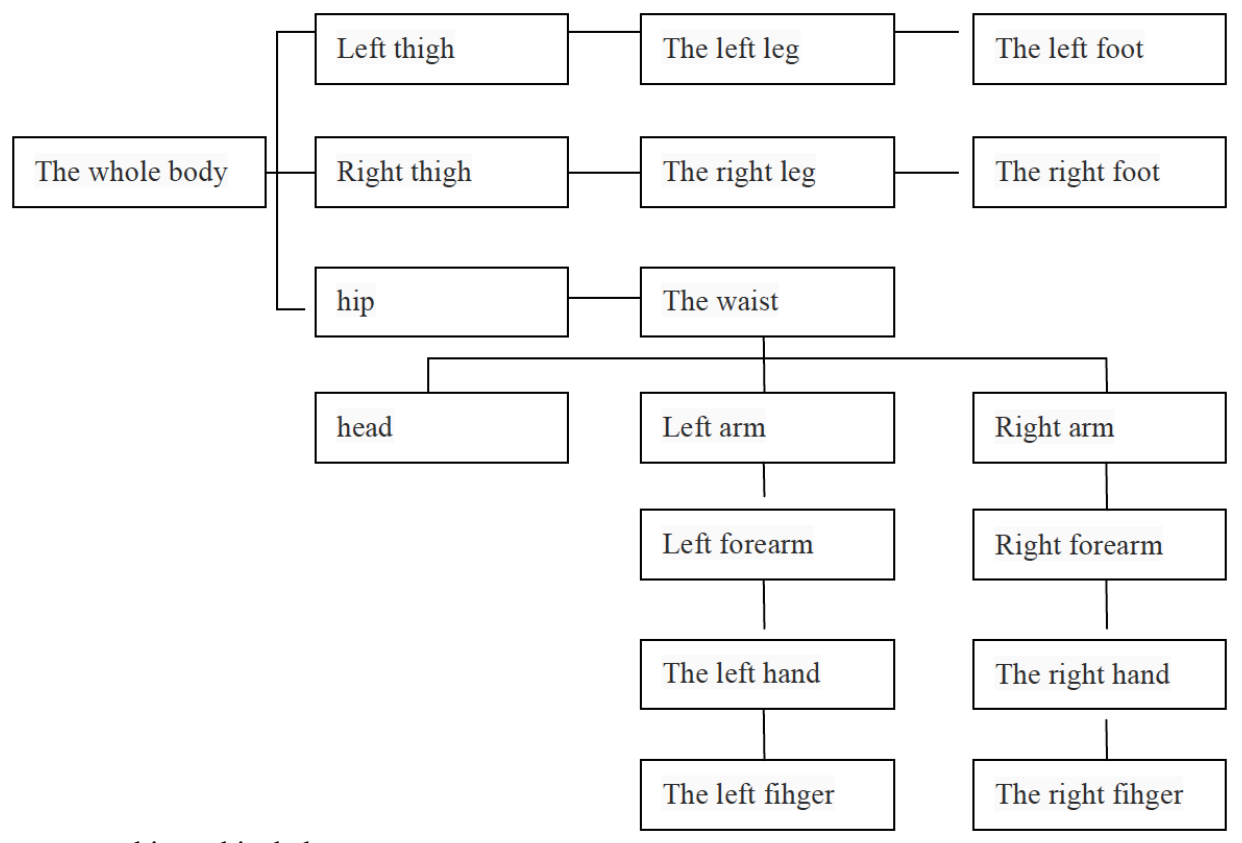

Fig. (1). Human motion neuron hierarchical chart.

Table 1. Technology and method for two types of artificial intelligence.

\begin{tabular}{|c|c|}
\hline Artificial Intelligence Technology & Method \\
\hline Fuzzy mathematical technology & $\begin{array}{l}\text { Adopting fuzzy mathematical method to eliminate interference factor and secondary factor in processing of } \\
\text { sports motion to determine motion fuzzy characteristic. }\end{array}$ \\
\hline Neural network technology & $\begin{array}{l}\text { Adopting self learning function of neural network to automatically extract and refine fuzzy rule from sports } \\
\text { motion image to set up the intelligence system for sports motion analysis and pattern recognition with adap- } \\
\text { tive capability. }\end{array}$ \\
\hline
\end{tabular}

$\mathrm{Z}(\mathrm{M})=<\mathrm{L} \alpha(\mathrm{M}), \mathrm{A}(\mathrm{M}), \mathrm{L} \beta(\mathrm{M}), \mathrm{S}(\mathrm{M})>$

It is observed from formula (1) that the characteristic $\mathrm{Z}(\mathrm{M})$ of sports motion $\mathrm{M}$ is expressed by special formula: $\mathrm{L} \alpha(\mathrm{M})$ is motion characteristic limb set, $\mathrm{A}(\mathrm{M})$ is motion characteristic, $L \beta(M)$ is state characteristic limb set, $S(M)$ is state characteristic.

$\mathrm{S}(\mathrm{M})=\{<\mathrm{Li}, \operatorname{Ti1}(\mathrm{M})>\mathrm{Li} \in \mathrm{L} \beta(\mathrm{M})\}$

It is observed from formula (2) that state characteristic $\mathrm{S}(\mathrm{M})$ is composed of limb $\mathrm{Li}$ in state characteristic limb set and its state characteristic Ti in first frame motion image and 1(M). The state characteristic of limb Li can be presented by arc value of included angle between limb and reference object (scene or upper limb) or by relative length (limb length is unit 1) between limb distal end and reference object (scene or upper limb joint). Quantitative analysis in sports contains two types of artificial intelligence technologies, and the methods are as follows:

\section{PATTERN RECOGNITION METHOD FOR SPORTS MOTION BASED ON NEURAL NETWORK TECHNOLOGY}

Pattern recognition is carried out for the two sports motions to better compare the motions and determine the match and mismatch and analyze the difference [4] between matching degree and motion data.

\subsection{Comparative Method For Sports Motion Patterns}

Suppose the standard motion is $\mathrm{M}$ and the comparative motion is $\mathrm{X}$ and (D1.....Ds) is weighting coefficients to express the extent of influence of every motion point on entire body, suppose $<\mathrm{L} 1(\mathrm{M}, \mathrm{X}), \ldots \ldots \mathrm{Ls}(\mathrm{M}, \mathrm{X})>$ is calculation formula for matching and membership function for a group of motion points. The matching and membership function between motion $\mathrm{X}$ and standard motion $\mathrm{M}$ is $\mathrm{L}(\mathrm{M}, \mathrm{X})$ :

$\mu(\mathrm{M}, \mathrm{X})=\sum_{\mathrm{j}=1}^{\mathrm{s}} \delta \mathrm{j} \times \mathrm{Lj}(\mathrm{M}, \mathrm{X}) / \sum_{\mathrm{j}=1}^{\mathrm{s}} \delta \mathrm{j}$

The threshold value of matching and membership of set pattern is $G$, when the matching and membership $L(M, X)$ for motion pattern is more than $\mathrm{G}, \mathrm{X}$ matches with $\mathrm{M}$, otherwise, they are mismatched.

\subsection{Neural Network Structure}

Neural network is an artificial intelligence technology to simulate brain work, mainly characterized by complex parallel mechanism and automatic learning function to obtain relevant knowledge about the motion pattern by quantitative analysis of sports motion so as to ensure its broader application value. Currently, the neural network structure in common use contains three aspects, the first is input layer. Relevant personnel can compare difference between motions based on data on motion and then match the rule according 


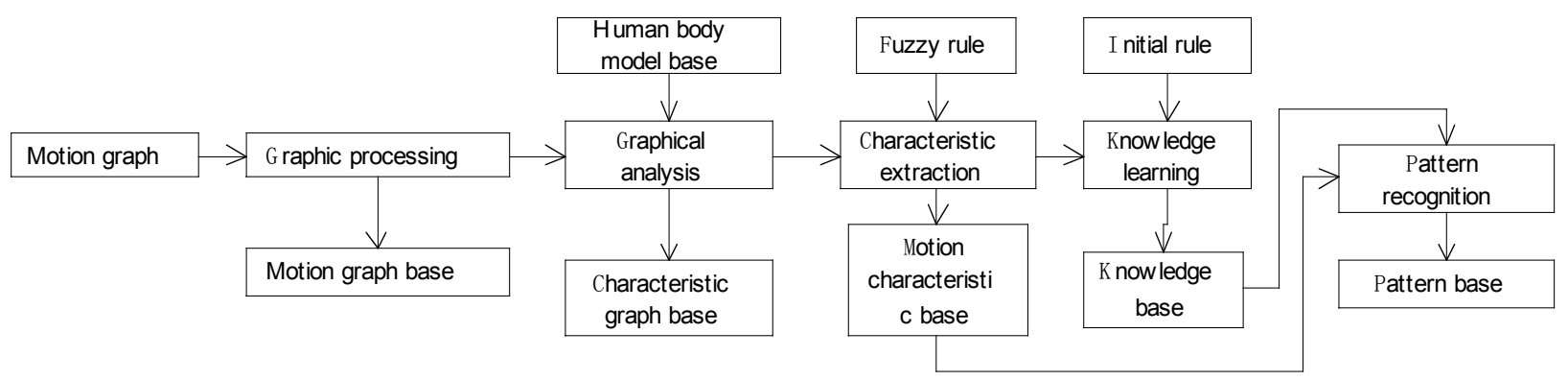

Fig. (2). System function flow chart.

Table 2. Function and purpose of the system.

\begin{tabular}{|c|c|}
\hline Classification of Functions & Purpose \\
\hline Graphical analysis & $\begin{array}{l}\text { To carry out view rectification to make motion images into front view. Graph refining processing makes stereogram for } \\
\text { motion into graph for motion. The graph for motion is abstracted into sports motion characteristic sketch according to } \\
\text { hierarchical model for human body. }\end{array}$ \\
\hline Graphical processing & $\begin{array}{l}\text { To input sports motion images into computer and make them have uniform file format, attribute and graphic specifica- } \\
\text { tion, carry out graphic repair to remove noise and irrelevant scene and encode motion image and send it into base. }\end{array}$ \\
\hline Characteristic extraction & $\begin{array}{l}\text { Limb set in motion, limb set in state and their motion characteristic data are obtained according to sports motion charac- } \\
\text { teristic sketch and fuzzy rule. }\end{array}$ \\
\hline Knowledge learning & $\begin{array}{l}\text { For standard sports motion, its motion pattern parameter and rule can be obtained by learning mechanism for fuzzy- } \\
\text { neural network and input the obtained knowledge into knowledge base. }\end{array}$ \\
\hline Pattern recognition & $\begin{array}{l}\text { To compare the given sports motion with the designated standard motion to obtain its motion pattern matching and } \\
\text { discrepant data in motion. }\end{array}$ \\
\hline
\end{tabular}

to experts' opinion on modification and ensure neural network is in proper operation. The second is hidden layer. Relevant personnel can learn motion pattern in the process and then select proper matching degree for motion according to self condition; and the third is output layer, during which comparative result [5] is obtained.

\subsection{Learning Method For Neural Network}

For the new standard sports motion, relevant personnel shall carry out learning based on neural network to obtain detailed content of motion pattern. Relevant personnel shall select motion pattern based on such knowledge by the following means:

1. To adjust the rule and content prepared beforehand according to relevant rules so as to ensure their initialization pattern;

2. Relevant personnel shall understand standard motion and its matching based on relevant content of system so as to know values of ultimate motion and then transform it into basic sample motion graph [6] according to the condition.

3. Relevant personnel shall carry out adjustment and regulation based on comments of sports experts and then input the proper result in the system.

Relevant personnel, during operation of neural network, shall understand four points: the first is motion point weighting $\delta 1 \ldots \delta \mathrm{s}$. The second is component motion (including 4 components, namely time, speed, amplitude and direction) weighting coefficient $\eta 1 \ldots \eta 4$. The third is adjustment coef- ficient $\Phi 1 \ldots \Phi 5$ for degree of membership of component motion at motion point; the fourth is membership function $\mu \mathrm{j} 1 \ldots \mu \mathrm{j} 4$ at limb motion point in initial motion.

For limb Li in motion, its time, speed, amplitude and direction in motion at motion point $\mathrm{Z}(\mathrm{X})$ is subject to initial expression of degree of membership $\mathrm{Lj}, 1 \ldots \mathrm{Lj}, 4$ of standard motion M:

$$
\begin{aligned}
& \mu \mathrm{j} 1(\mathrm{X}, \mathrm{M})=1-\frac{\text { fkeyi }(\mathrm{M})-\mathrm{fkeyi}(\mathrm{x})}{\mathrm{ml}} \times \Phi 1 \\
& \mu \mathrm{j} 2(\mathrm{X}, \mathrm{M})=1-\frac{\Delta \mathrm{f}(\mathrm{M}) \Delta \mathrm{fi}(\mathrm{X})}{\mathrm{m}} \times \Phi 2
\end{aligned}
$$

\section{SYSTEM FUNCTION AND PROCESSING FLOW}

Quantitative analysis and pattern recognition system for sports motion contain five processes namely graphic processing, graphical analysis, motion characteristic extraction, pattern recognition, knowledge learning and motion pattern as shown in Fig. (2).

It is observed from Fig. (2) that every function in the system plays a key role and ensures quantitative analysis for sports motion is more efficient [7]. Based on the processing and analysis by the system, the detailed functions and purposes thereof are as shown in Table $\mathbf{1}$.

Design and implementation of intelligent system for quantitative analysis of sports motion can determine motion and training and keep the training process in the form of im- 
age video for reference in physical training later. Quantitative analysis of sports motion is the method commonly used in international sports, and conducts qualitative and quantitative analysis of athletes. This is the embodiment of the application of modern technology in physical training.

The paper takes video image for sports motion as primary reference for research on athletes, which can implement video control under technical guidance for montage of the collected images. The moving object under tracking can bring quantitative function into play and the established analysis system for sports motion is conducive to improving quantitative analysis effect for sports motion. Traditionally, the design of sports motion quantitative system is mainly done by manual observation and analysis, which has obvious limitations in application.

Analysis of athletes by intelligent system for sports motion quantization is able to implement effective combination of information, time and space and serve as scientific guidance for athlete training under the guidance of kinesiology parameter and ensure improvement in athlete's ability and implementation of quantization and intelligentization of sports motion. The paper presents a video-based sports motion analysis system studied and designed according to Visual $\mathrm{C}++$. net 2003 development platform to implement qualitative and quantitative analysis of motion video, which is of great practicability. The paper presents human motion analysis based on monocular video, control of motion video according to Directshow technology, extraction of background information from video, time domain segmentation of video image, extraction of moving object from video to synthesize a panoramic picture adopting image mosaicking technology. Besides, it filters static background by interframe difference method to realize background suppression and carries out automatic tracking of key moving part based on Kalman filtering and color block matching technology, and also obtains kinesiology parameter and set up videobased sports motion analysis system.

\section{CONCLUSION}

The above mentioned sports motion quantitative analysis together with elaboration of design and implementation of intelligent system enable relevant personnel to adopt two feasible artificial intelligence technologies to better carry out quantitative analysis of sports motion and improve motion and facilitate smooth integration of motion image and motion point.

Neural network is an artificial intelligence technology simulating brain work, characterized by complex and parallel mechanism and automatic learning function. During the quantitative analysis on sports motion, the self learning function of neural network can be used to obtain the relevant knowledge of comparison of motion patterns, and then its application function is broadened.

Accordingly, relevant personnel shall select applicable pattern and technical means for key analysis based on practical situation to offer more convenience for research and expedite analysis to improve system design and satisfy many requirements of functions.

\section{CONFLICT OF INTEREST}

The author confirms that this article content has no conflict of interest.

\section{ACKNOWLEDGEMENTS}

Declared none.

\section{REFERENCES}

[1] X. Zhao, H. Zhang, and J. Lu, "Solution to Business Intelligence Application System of Large Iron and Steel Group," Computer Integrated Manufacturing System, no. 9, pp. 104-105, 2010. http://en.cnki.com.cn/Article_en/CJFDTOTAL-JSJJ201009030.htm

[2] F. Xiong, "System Architecture and Implementation of Intelligence System Technique towards Agriculture Agricultural Field," Pattern Recognition and Artificial Intelligence, no. 5, pp. 158-159, 2012.

[3] S. Wei, "Design of Multimedia Intelligence System Solution for Communities," Journal of Shijiazhuang University, no. 6, pp. 137$138,2012$.

[4] J. Wang, and L. Zhou, "Design and Implementation of Building Intelligence System based on Internet of Things," Digital Technology \& Application, no. 7, pp. 169-170, 2013.

[5] C. Li, and W. Zhou, "Analysis on Physical Intelligence and its Project," Contemporary Sports Technology, no. 27, pp. 119-120, 2012.

[6] L. Chen, G. Li, P. Wang, and L. Zhou, "Design and Implementation of Intelligence System for Machine Room," Chongqing Medicine, no. 35, pp. 241-242, 2011.

[7] X. Miao, "Design and Implementation of Multimedia Data Base System for Physical Standard Motions," Sports Science, no. 3, pp. 129-130, 2010.

\footnotetext{
Received: June 10, 2015

Revised: July 29, 2015

Accepted: August 15, 2015

(C) Wang Ligang; Licensee Bentham Open.
}

This is an open access article licensed under the terms of the (https:/creativecommons.org/licenses/by/4.0/legalcode), which permits unrestricted, noncommercial use, distribution and reproduction in any medium, provided the work is properly cited. 